\title{
Novel Multi-Functional Correlative Neural Tract Tracers for Elucidating the Developing Nervous System in Thick Biological Specimens Using Wide-Field Fluorescence 3-D Deconvolution Microscopy
}

\author{
Rosa-Molinar, E. and J. L. Serrano-Velez ${ }^{* * *}$ \\ *Biological Imaging Group, University of Puerto Rico-Rio Piedras, San Juan, PR 00931-1809, * Julio Garcia \\ Diaz Center for Investigations in Biology, University of Puerto Rico-Rio Piedras, San Juan, PR 00931-1809.
}

In response to neuroembryologists' and neuroanatomists' need for sophisticated tools to trace neural pathways and circuits [1], in recent years we have seen an explosion of fluorescent probes and methods that allows visualization of the intricate structural details of living (dynamic) and fixed (static) individual and/or groups of neurons in the brain and/or spinal cord. Golgi staining, developed by Camillo Golgi and elevated to an art form by Santiago Ramon y Cajal over a century ago [2], is the classic but time consuming method of visualizing the intricate structural details of individual and/or groups of neurons in the brain and/or spinal cord, and it is still in wide use today. During neurophysiological experiments, it is possible to identify individual and/or groups of neurons in the brain and spinal cord by iontophoretic filling with fluorescent tracers. Another method of achieving visualization and identification of structures uses gene gun-mediated ballistic delivery of gold or tungsten particles carrying cDNAs (Biolistics), lipophilic dyes (DiOlistics), or fluorescent intracellular calcium indicators (Calistics) into the brain and or spinal cord [3]. These advances have moved in parallel with improvements in visualization technology such as wide-field fluorescence 3-D deconvolution microscopy that generates a stack of fluorescent images at different focal planes and then employs a set of sophisticated algorithms to determine point-spread functions for sources of fluorescence in thick biological specimens that, in turn, enhance the ability to visualize and measure low light intensity signals from a small number of fluorescent dye molecules in living and fixed individual and/or groups of neurons within the brain and/or spinal cord [4].

Understanding the nervous system and gaining insight into the complex intercellular mechanisms, processes, and interactions during post-embryonic development is one of the primary goals of my research program. Experimental models provide the basis for much of the research; for example, the Western Mosquitofish, Gambusia affinis affinis, provides a versatile experimental model [5]. However, a major restriction to realizing the full potential of this vertebrate model system, particularly with regard to understanding development and differentiation of the nervous system, is the lack of sufficiently refined and precise neural tract mapping techniques and probes.

We describe our work on developing and characterizing novel multi-functional correlative neural tract tracers prepared by the conjugation of mono-sulfo-NHS- Nanogold $₫$ to 10,000 molecular weight (MW) aminofunctionalized dextran conjugated to fluorescein [Nanogold FDA] and Texas Red ${ }^{\circledR}$ [Nanogold TDA] (see Figure $1 \mathrm{a}$ and $1 \mathrm{~b}$ ). We use these multi-functional correlative neural tract tracers to retrogradely fill ventral horn spinal motor neurons in the lateral motor column innervating the musculature of the sexually dimorphic median unpaired fin, the gonopodium in male G. a. affinis [5].

Small strips of nylon filter-paper-coated Nanogold FDA implanted into the epaxialis musculature retrogradely filled a ventromedial motor column of large unipolar spinal motor neurons (mean of $16.3 \mu \mathrm{m}$ ) ventromediolateral to the central canal of the spinal cord (see Figure 1a).

Small strips of nylon filter-paper-coated Nanogold TDA implanted into the sexually dimorphic musculature of the gonopodium labeled a ventrolateral spinal motor column of smaller multipolar spinal motor neurons (means range from 7.7 to $11.9 \mu \mathrm{m}$; see Figure $1 \mathrm{~b}$ ). The extensive intermingling of the cell bodies and overlap of the dendritic fields among the ventrolateral spinal motor column of smaller multipolar spinal motor neurons suggest that subregions of the motor neuronal pool may participate in coordinating the erector and depressor muscles of the musculus erector analis major (mEAM). The ventrolateral spinal motor column of smaller multipolar spinal motor neurons projecting to the erector and depressor muscles of the mEAM are distributed in spinal cord segments associated with trunk vertebrae 8-16 (Tk8-16). 
Nanogold TDA and Nanogold FDA required approximately $8 \mathrm{~h}$, yielding dye diffusion rates of $1.0 \mathrm{~mm} / \mathrm{h}$ or more, respectively. This contrasts with other neural tract tracers such as the lipophilic dye Di-I, silver impregnation, and horseradish peroxidase in which diffusion rate are typically $0.003 \mathrm{~mm} / \mathrm{h}$ or less, and the distance traversed amounts to only a few mm even after months of transport. Using Nanogold TDA and Nanogold FDA combined with wide-field fluorescence 3-D deconvolution microscopy (see Figure 1c) and transmission electron microscopy we have identified the presence of a new sexually dimorphic spinal nucleus in the spinal cord of a teleost fish.

\section{References:}

[1] Kiernan, J. A. Barr's The Human Nervous System An Anatomical Viewpoint, Lippincott Williams and Wilkins, Eighth Edition, 2004.

[2] Ramon y Cajal, S. Histology of the Nervous System of Man and Vertebrates. Translated from the French by N. Swanson and L. Swanson, 1995.

[3] Gan, W. B., et al. Multicolor "DiOlistic" Labeling of the Nervous System using Lipophilic Dye Combinations. Neuron 27(2):219-25, 2000.

[4] Wallace, W., et al. A Workingperson's Guide to Deconvolution in Light Microscopy. BioTechniques 31(5): 1075-1097 (2001).

[5] Rosa-Molinar, E. Proc. of the First International Symposium on Livebearing Fishes, New Life Publications, Inc., Homestead, Florida (book chapter in press) 2005.

[6] Research supported through NSF/IBN-0091120 and U54-NS39405-06 to ER-M.

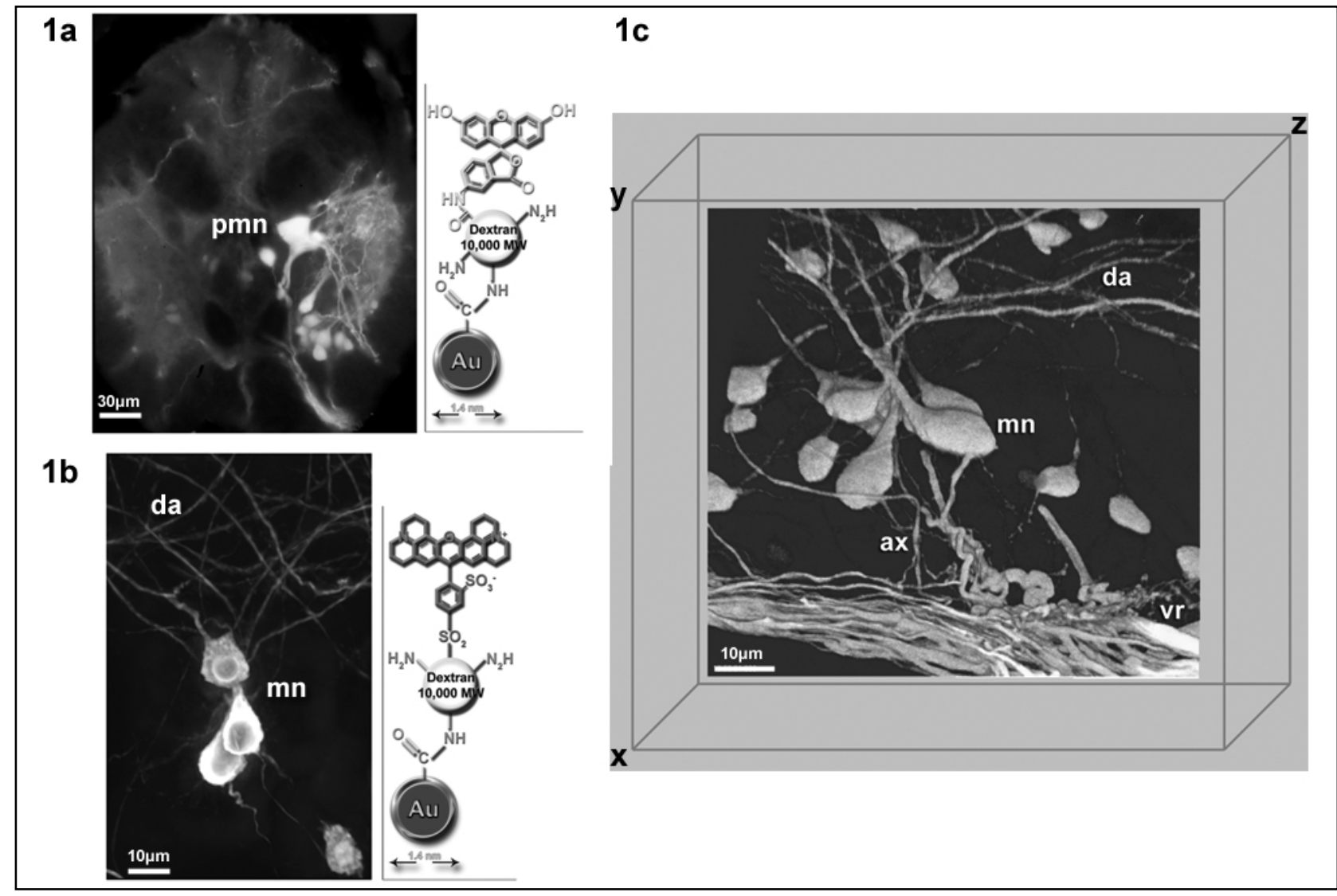

Figure 1. Photomicrographs of the epaxialis musculature motor neurons (1a; known as primary motor neurons [pmn]) retrogradely filled with Nanogold FDA (see inset 1a). m. erector analis major motor neurons [mn] (1b) retrogradely filled with Nanogold TDA (see insert 1b). Note the fan shape radiation of the dendritic arbor (da; see Figure 1b). 3D-reconstructed $m$. erector analis major motor neurons [mn; see Figure 1c], axons [ax], ventral root [vr], and dendritic arbors [da] within a $30 \mu \mathrm{m}$ whole-mount preparation. 